\title{
Abdelmageed Abdelrahman MBBCh BAO DIPM DFSRH MRCOG MSc (clinical education)
}

\author{
Abdelmageed Abdelrahman ${ }^{1}$ \\ Received: 22 March 2021 / Accepted: 26 April 2021 / Published online: 17 May 2021 \\ (C) The International Urogynecological Association 2021
}

\section{Commentary on 'Timing of recurrence after surgery in pelvic organ prolapse'}

This observational cohort study aimed to determine when women are at risk for recurrent pelvic organ prolapse. The study was conducted in a Dutch hospital using a routine clinical care database. Inclusion criteria were symptomatic prolapse (bulging complaints or dragging sensation), clinically relevant prolapse (defined as prolapse in at least one compartment of POP-Q stage 2 or higher) and underwent prolapse surgery. Exclusion criteria included women within 6 months of delivery or if they were aged younger than 18 years. The primary outcome measure was prolapse recurrence.

Results showed that 46 out of 274 women had prolapse recurrence during a mean follow-up time of $55 \pm 32$ months. Recurrence of any compartment was most likely in the first 2 years after prolapse surgery. In years 3 and 4 this increase was still present but less steep, remaining stable thereafter. The risk of recurrence seems largest when all three compartments are involved. The authors concluded that the risk of recurrence is different according to the type of prolapse surgery and that over time recurrence did not differ for prolapse repair with vaginal hysterectomy or sacrospinous ligament fixation.

To our knowledge, this is the first study showing the recurrence over time for the posterior and apical compartment in one of the largest Dutch datasets collected to date. Limitations of the study include no standardized definition for surgical success following prolapse surgery and detection bias as women who did not visit the outpatient clinic were assumed to have no recurrence. In addition, the number of women in some categories was small.

\section{Declarations}

Conflict of interest None.

Publisher's note Springer Nature remains neutral with regard to jurisdictional claims in published maps and institutional affiliations.

Abdelmageed Abdelrahman

abdelmageed@hotmail.co.uk

1 Department of Urogynaecology, Liverpool Women's Hospital NHS Foundation Trust, Liverpool, UK 